\title{
Searches for squarks and gluinos in events with missing transverse momen- tum
}

\author{
Alessandro Gaz ${ }^{1, a}$ \\ ${ }^{1}$ University of Colorado, \\ on behalf of the ATLAS and CMS Collaborations
}

\begin{abstract}
In this contribution, the latest results from CMS and ATLAS on inclusive searches for Squark and Gluino Production at the LHC are reviewed. A variety of complementary final state signatures and methods are presented using up to $20 \mathrm{fb}^{-1}$ of data from the $8 \mathrm{TeV}$ LHC run of 2012. Interpretations of the experimental results in SUSY models are covered with a special emphasis on final states with jets, photons, and at most one lepton.
\end{abstract}

\section{Introduction}

The observation [1] of a new particle of mass $\sim 125 \mathrm{GeV}$, whose properties are consistent with those expected for the standard model Higgs boson poses new challenges to modern particle physics. It is expected that the mass of the Higgs boson (not being protected by any fundamental symmetry) will receive large corrections from the interaction with higher-scale particles. In order to constrain the mass of the Higgs boson to the observed value, it is necessary to postulate either an unrealistic fine-tuning of the parameters or a mechanism that can protect the Higgs mass to go much above the scale of the electroweak symmetry breaking. One of the most popular such mechanisms is that provided by supersymmetry, in which additional particles provide corrections that cancel almost exactly the contributions from the standard model particles. In particular, natural supersymmetry (see e.g. [2]) is a very appealing scenario; in this framework the masses of the supersymmetric partners of the top and bottom quarks $(\tilde{t}, \tilde{b})$ are expected not to exceed $O(500 \mathrm{GeV})$, and the masses of the superpartners of gluons $(\tilde{g})$ cannot be much heavier than $1 \mathrm{TeV}$. In this scenario, the ATLAS and CMS experiments, with the $2012 \sqrt{s}=8 \mathrm{TeV}$ dataset, are already in a position to obtain evidence of new physics phenomena or exclude a large fraction of the natural or other SUSY models.

\section{Searches for squarks and gluinos in events with missing transverse energy}

The ATLAS and CMS detectors are described in detail elsewhere [3]. In all the analyses summarized here, it is assumed $R$-parity conservation, so the lightest supersymmetric particle (LSP) is stable and traverses the detector

\footnotetext{
a e-mail: alessandro.gaz@ cern.ch
}

unseen. The dominant signature for such kind of events is thus a large amount of missing transverse energy (MET). Other variables extensively used in the analyses are the number of $(b$-)jets, the scalar sum of the transverse momentum of the jets $(H T)$, and the sum of MET and $H T$ $\left(m_{\text {eff }}\right)$. All the analyses use part or whole the $2012 \sqrt{s}=8$ $\mathrm{TeV}$ dataset, whose integrated luminosity is about $20 \mathrm{fb}^{-1}$.

\subsection{CMS: photons}

CMS uses $4.04 \mathrm{fb}^{-1}$ to perform a search for events with one or two high- $p_{T}$ photons, hadronic jets and MET [4]. This search targets General Gauge Mediated (GGM) scenarios, in which the LSP is a very light gravitino $(\tilde{G})$, and the neutralino is the next-to-lightest SUSY particle. In the cases in which the neutralino (produced in the LHC environment by the decay of gluinos and squarks) is mostly bino-like, its dominant decay is $\chi_{1}^{0} \rightarrow \gamma \tilde{G}$.

The analysis proceeds in two streams: a single photon and a di-photon search. In the first case the presence of a photon candidate with $p_{T}(\gamma)>80 \mathrm{GeV}$, along with at least two hadronic jets with $p_{T}($ jet $)>40 \mathrm{GeV}$ (and $H T>450 \mathrm{GeV}$ ) is required. In the second, two photons with $p_{T}\left(\gamma_{1}\right)>40$ and $p_{T}\left(\gamma_{2}\right)>25 \mathrm{GeV}$ and at least one hadronic jet with $p_{T}(j e t)>30 \mathrm{GeV}$ are selected.

Backgrounds arise from multijet (QCD) events in which the MET originates from the mismeasurement of the $p_{T}$ of one or more jets, and from electroweak events containing real $M E T$, in which an electron has been misidentified as a photon. The first is estimated using data control samples with loosened photon-ID requirements, for the second, the $\gamma$ mis-identification probability is measured using ee and $e \gamma$ samples. Remaining small contributions are taken from the simulation.

No significant excess is observed over the expected standard model backgrounds. Limits are set in the con- 


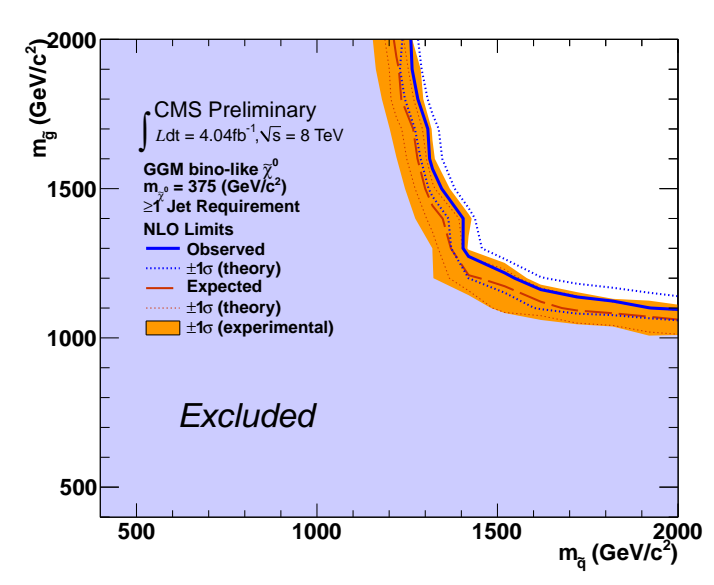

Figure 1. Exclusion plot for the CMS di-photon analysis in the $m_{\tilde{g}}$ vs $m_{\tilde{q}}$ plane. It is assumed that the mass of the neutralino is $375 \mathrm{GeV}$.

text of the GGM model, for both bino-like and winolike neutralinos (in the second case the dominant decay is $\left.\chi_{1}^{0} \rightarrow Z \tilde{G}\right)$, considering a neutralino of mass $375 \mathrm{GeV}$. The single- and di-photon analyses have similar sensitivity and can exclude, in the most favorable case, masses of both gluino and squarks above $1 \mathrm{TeV}$. Fig. 1 shows the 95\% CL exclusion plot for the di-photon analysis in the bino-like scenario.

\subsection{ATLAS: jets $+\tau$ 's}

ATLAS uses their full 2012 dataset (corresponding to 20.7 $\mathrm{fb}^{-1}$ ) to search for SUSY signatures with at least one $\tau$ lepton in the final state [5]. The analysis is optimized for models in which the $\tilde{\tau}$ is the next-to-lightest SUSY particle, which decays predominantly to $\tau$ and gravitino.

It is required the presence of at least two hadronic jets (with $p_{T}\left(\right.$ jet $\left._{1}\right)>130$ and $p_{T}\left(\right.$ jet $\left._{2}\right)>30 \mathrm{GeV}$ ) and $M E T$ $>150 \mathrm{GeV}$. Different selections are then considered in order to maximize the sensitivity to different models. Only hadronic decays of $\tau$ candidates are considered, and either one candidate with medium $\tau$-ID tightness and no additional $\tau$ candidates, or two loose $\tau$ 's are selected.

The main backgrounds arise from $t \bar{t}, W$ and $Z+$ jets events with either real or fake $\tau$ candidates. These are estimated from data control regions and the predicted yield in the signal regions is estimated by means of scaling factors derived from the simulation.

The observed yields are in good agreement with the predicted background. Limits are set in the context of Gauge Mediated Supersymmetry Breaking (GMSB) and Natural Gauge Mediated Model (nGM). In both cases masses of gluinos below $1 \mathrm{TeV}$ are excluded and, as can be seen from Fig. 2, the limits are practically independent of the mass of the $\tilde{\tau}$.

\subsection{ATLAS: same sign leptons}

The full ATLAS $8 \mathrm{TeV}$ sample $\left(20.7 \mathrm{fb}^{-1}\right)$ is then used to search for the production of squarks and gluinos that

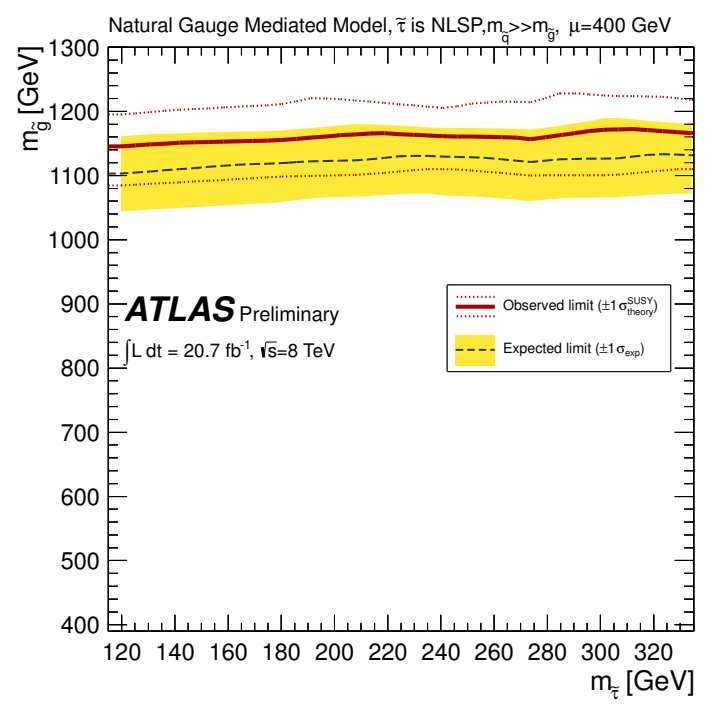

Figure 2. ATLAS exclusion limits (at 95\% CL) on the mass of the gluino as a function of the mass of the stau, in the context of the nGM model.

originate final states with two same-sign lepton (electrons or muons) candidates [6].

The standard model predicts very low background yields for this kind of final states, mostly arising from $t \bar{t} V$ and $V V$ events, where $V$ is either a $W$ or a $Z$ boson; these contributions are taken from the Monte Carlo. Other background sources include charge mis-measurement of electrons irradiating a photon, and leptons associated to $b$-jets that are not rejected by the isolation criteria. These contributions are estimated with data-driven methods.

Events with two same-sign leptons ( $e$ 's or $\mu$ 's) are selected, requiring the $p_{T}$ of the lepton candidates to be above $20 \mathrm{GeV}$, and $|\eta|<2.47$ (2.40) for $e$ 's ( $\mu$ 's). Events are then categorized based on the number of reconstructed (b)-jets. The minimum $p_{T}$ for the generic hadronic jet is $40 \mathrm{GeV}$; this threshold is lowered to $20 \mathrm{GeV}$ for the $b$-jets candidates, in order to be sensitive to scenarios with soft $b$-jets in the final states.

No significant excess is observed above the standard model predictions, and exclusion limits are set for a variety of models. Fig. 3 shows the regions (in the $m_{\tilde{\chi}_{1}^{0}}$ vs $m_{\tilde{q}}$ plane) excluded at $95 \% \mathrm{CL}$ for direct squark production, followed by decays through sleptons.

\subsection{CMS: $\alpha_{T}$}

The CMS Collaboration uses $11.7 \mathrm{fb}^{-1}$ of the $\sqrt{s}=8 \mathrm{TeV}$ dataset to search for SUSY signatures using the variable $\alpha_{T}$ [7]. For di-jet events, $\alpha_{T}$ is defined as:

$$
\alpha_{T}=\frac{E_{T}^{j_{2}}}{M_{T}}
$$

where $E_{T}^{j_{2}}$ is the transverse energy of the less energetic jet and $M_{T}$ is the transverse mass of the di-jet system. This definition is generalized for multijet events by combining 


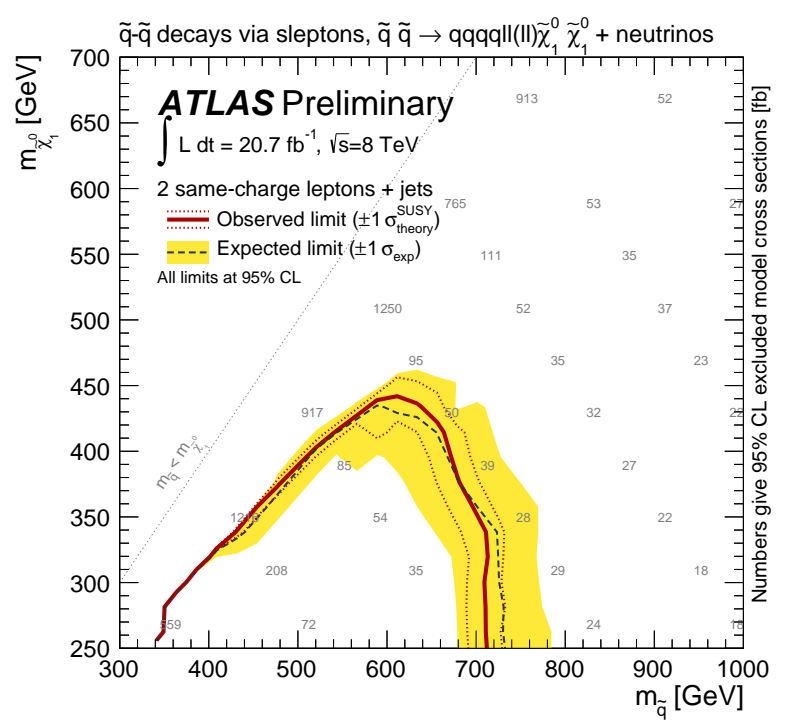

Figure 3. ATLAS limits on direct squark production (and subsequent decay of the squarks through sleptons) from the analysis with same-sign leptons in the final state.

the jets into two pseudo-jets; the chosen combination is the one that minimizes the difference in transverse energy of the two pseudo-jets. By construction $\alpha_{T}$ is very robust against jet energy mismeasurements that can fake missing transverse energy. QCD multijet events (with no real $M E T)$ exhibit an $\alpha_{T}$ distribution that has a natural cut-off at $\alpha_{T} \sim 0.5$. Events with $\alpha_{T}>0.55$ are characterized by genuine sources of $M E T$.

The analysis thus selects events with $\alpha_{T}>0.55$ and $H T>275 \mathrm{GeV}$ : QCD multijet events passing this cut are suppressed to a negligible level and the dominant background is constituted by $t \bar{t}$, single $t$, and $W, Z+$ jets events. The sample is split into two jet-multiplicity bins: $2 \leq n_{\text {jets }} \leq 3$ and $n_{\text {jets }} \geq 4$, and these samples are further split into eight bins of $H T$ and five bins of $b$-jet multiplicity $\left(n_{b-\text { jets }}=0,1,2,3\right.$, and $\left.n_{b-\text { jets }} \geq 4\right)$.

The backgrounds are determined from several data control samples (binned in the same way as the main signal sample): the $\mu+$ jets sample is used to estimate the $t \bar{t}$, single $t, W+$ jets, and $Z+$ jets for $n_{b-\text { jets }} \geq 2$, while the $\mu \mu$ + jets and $\gamma+$ jets samples are used to determine the $Z+$ jets background for $n_{b-j e t s}<2$. The background contributions into the signal bins are calculated by multiplying the event yields of the corresponding data control samples by some translation factors derived from the simulation:

$$
N_{\text {pred }}^{\text {signal }}=\frac{N_{M C}^{\text {signal }}}{N_{M C}^{\text {control }}} \times N_{o b s}^{\text {control }} .
$$

Several closure tests on the data are performed in order to check the consistency of the procedure and to assign an appropriate systematic uncertainty.

No significant excess is observed in the data, compared to the background predictions. Exclusion limits, in terms of the simplified model in which gluino pairs are produced

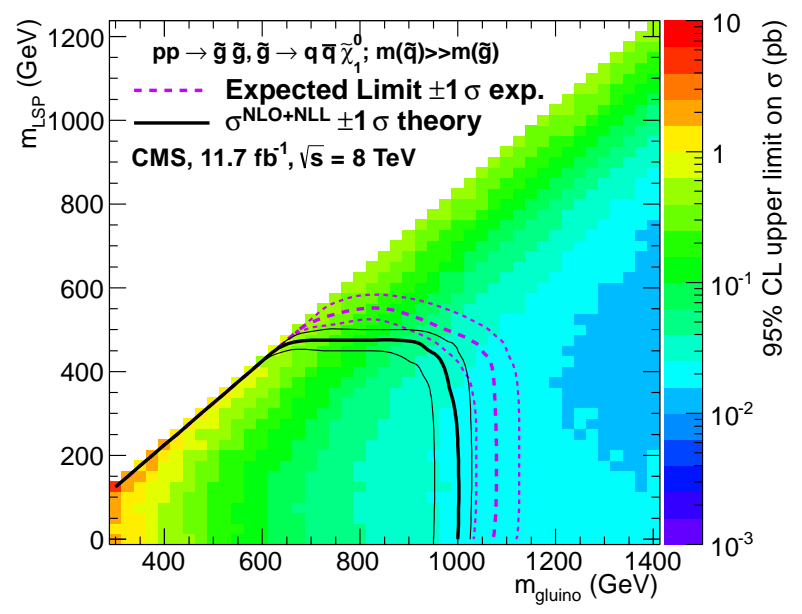

Figure 4. Exclusion limits for the CMS $\alpha_{T}$ analysis for the simplified model in which gluino pairs are produced and each gluino decays: $\tilde{g} \rightarrow q \bar{q} \tilde{\chi}_{1}^{0}$. The color scale indicates the observed crosssection $95 \%$ CL upper limit, and the lines indicated the observed (black) and expected (red) exclusion limits.

and each gluino decays to a pair of light $q \bar{q}+$ the LSP, are presented in Fig. 4.

\subsection{ATLAS: MET + jets}

The full dataset $\left(20.3 \mathrm{fb}^{-1}\right)$ collected by the ATLAS experiment is used to search for new physics phenomena in final states with jets and missing transverse energy [8]. Events with at least two jets, with $p_{T}\left(\right.$ jet $\left._{1}\right)>130 \mathrm{GeV}$ and $p_{T}\left(\right.$ jet $\left._{2}\right)>60 \mathrm{GeV}$ and are categorized into different signal regions based on the presence of additional jets with $p_{T}\left(\right.$ jet $\left._{i}\right)>60 \mathrm{GeV}$. The key selection variable is the effective mass $m_{e f f}$, for which two definitions are used:

$$
m_{e f f}=\sum_{N_{j e t s}} p_{T}\left(j_{i}\right)+M E T
$$

in which only the jets considered in the particular signal bin are used, and a more inclusive one, taking into account all the jets in the event with $p_{T}>40 \mathrm{GeV}$ :

$$
m_{\text {eff }}(\text { incl. })=\sum_{p_{T}\left(j_{i}\right)>40 G e V} p_{T}\left(j_{i}\right)+M E T .
$$

Selection requirements are imposed using both variables, and cuts are applied on the difference between the azimuthal angle of MET and that of the jets in the event, in order to suppress the QCD background.

Background arise from $W, Z+$ jets events, $t \bar{t}$, and QCD multijets. As in other analyses the contributions of these backgrounds into the signal regions are determined by multiplying the yields in data control samples by appropriate translation factors. The samples used in the analysis are multijet samples with reverted cuts on $\triangle \phi($ jet, $M E T)$ and on $M E T / m_{\text {eff }}$ (QCD enriched), $\gamma+$ jets (to determine the $Z+$ jets contribution), and a $\mu+$ jets sample, either $b$-vetoed (enriched in $W+$ jets) or $b$-tagged (enriched in $t \bar{t}$ ). The translation factors for the QCD background 


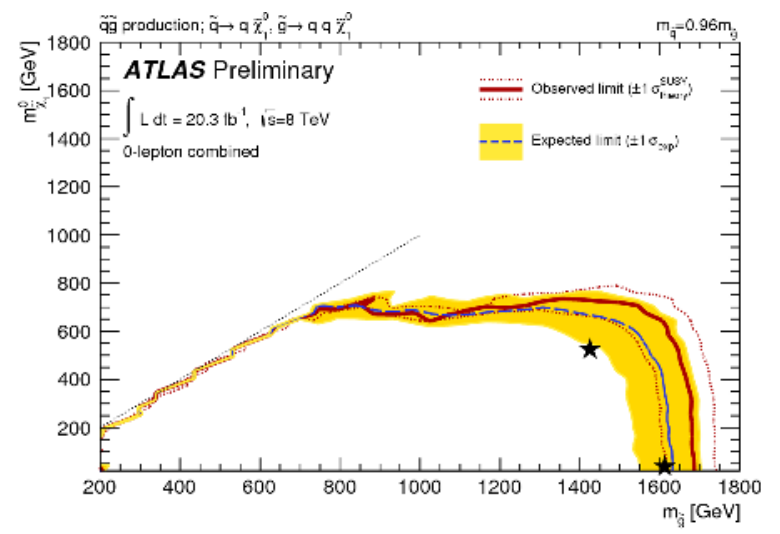

Figure 5. Exclusion limits set by the ATLAS $M E T+$ jets analysis on the squark-gluino production. The decay chains considered are $\tilde{q} \rightarrow q \tilde{\chi}_{1}^{0}$ and $\tilde{g} \rightarrow q \bar{q} \tilde{\chi}_{1}^{0}$, with the mass of $\tilde{q}$ being set to $96 \%$ of the mass of the $\tilde{g}$.

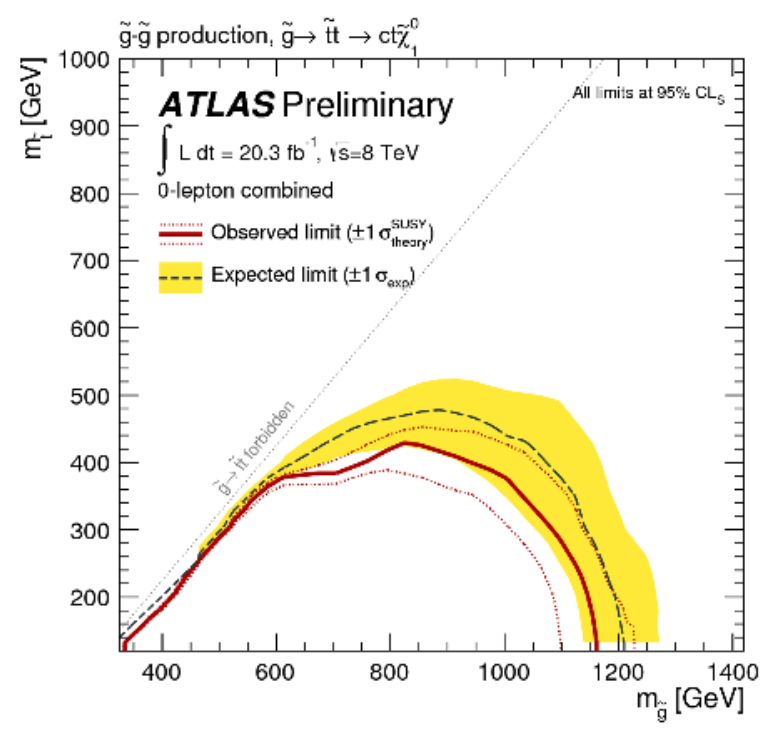

Figure 6. Exclusion limits set by the ATLAS $M E T+$ jets analysis on gluino-gluino production, with each gluino decaying to $t \overline{\bar{\chi}} \tilde{\tilde{\chi}}_{1}^{0}$.

are determined using a data-driven technique, applying a resolution function to well measured multijet events. For the other background categories, the translation factors are taken from the simulation.

No significant excess is seen compared to the background predictions, so limits are set for a variety of models. Fig. 5 shows the exclusion limits for squark-gluino production, where $\tilde{q} \rightarrow q \tilde{\chi}_{1}^{0}$ and $\tilde{g} \rightarrow q \bar{q} \tilde{\chi}_{1}^{0}$, with $m_{\tilde{q}}=$ $0.96 m_{\tilde{g}}$, while Fig. 6 considers $\tilde{g} \tilde{g}$ production, with $\tilde{g} \rightarrow$ $t \bar{t} \tilde{\chi}_{1}^{0}$.

\subsection{ATLAS: multijets}

Another ATLAS analysis, based on $20.3 \mathrm{fb}^{-1}$, looks for even higher jet multiplicities to search for SUSY signatures [9]. The analysis proceeds along two main streams.

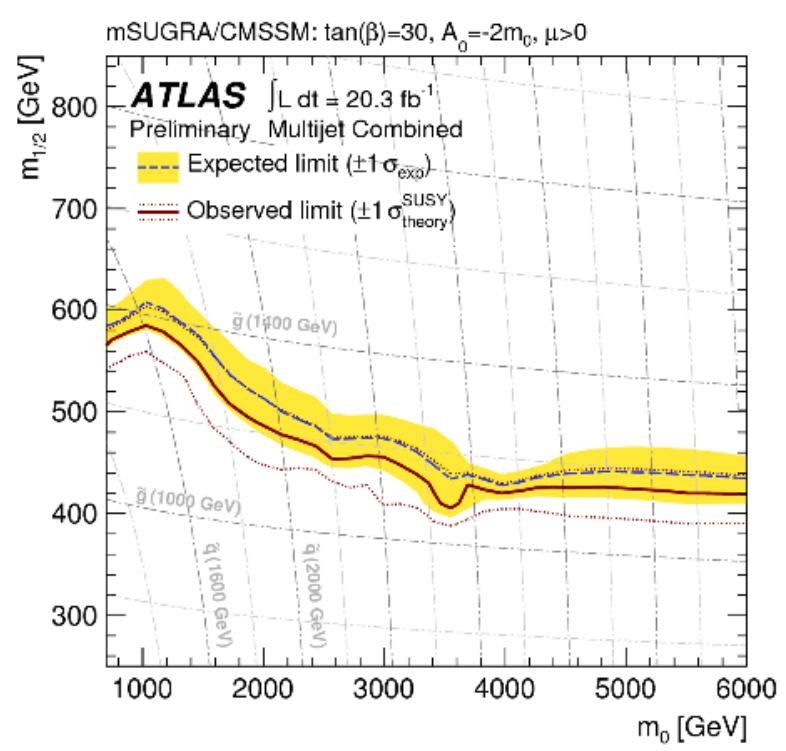

Figure 7. Exclusion limits of the ATLAS multijets analysis on the mSUGRA framework, with $\tan \beta=30$.

In the multijet + flavor stream, events with 8,9 , or $\geq 10$ jets with $p_{T}>50 \mathrm{GeV}$, or 7 or $\geq 8$ jets with $p_{T}>80 \mathrm{GeV}$ are selected into different signal regions. These samples are further split (except the $\geq 10$ jets bin), depending on their $b$-jet content $(0,1$, or $\geq 2 b$-jets).

In the multijet $+M_{J}^{\Sigma}$ stream, events with at least 8 jets with $p_{T}>50 \mathrm{GeV}$ and $|\eta|<8$ are selected. These jets are then used to feed the anti- $k_{t}$ clustering algorithm, setting the value of the radius parameter $R$ to 1.0 (the typical value for ATLAS analyses is 0.4). The selection variable $M_{J}^{\Sigma}$ is defined as:

$$
M_{J}^{\Sigma}=\sum_{j} m_{j}^{R=1.0},
$$

where the sum runs on the $R=1.0$ jets with $p_{T}^{R=1.0}>100$ $\mathrm{GeV}$ and $\left|\eta^{R=1.0}\right|<1.5$. A cut at 340 or $420 \mathrm{GeV}$ on $M_{J}^{\Sigma}$ is set, depending on the different signal regions considered.

The main selection variable in the analysis is the ratio $M E T / \sqrt{H T}$ (which is required to be $>4 \mathrm{GeV}^{1 / 2}$ for the signal regions). The estimate of the QCD multijet background relies on the observation that the shape of the $M E T / \sqrt{H T}$ distribution does not depend on the jet multiplicity of the event (there is some dependence though on the number of $b$-jets, so different $b$-jet multiplicities are considered separately). The QCD background is estimated from data control samples with lower jet multiplicity, extrapolating the number of expected events entering the signal region from the number of events with low $M E T / \sqrt{H T}$.

A $\mu+$ jets control sample is used to determine the $t \bar{t}$ and $W+$ jets background, using translation factors taken from the Monte Carlo. The $Z \rightarrow v \bar{v}$ component is predicted from the simulation after this has been validated using a $Z \rightarrow \mu^{+} \mu^{-}$data control sample.

No significant excess in seen over the background predictions in any of the different signal regions considered, so limits on different SUSY models are set. Fig. 7 shows 


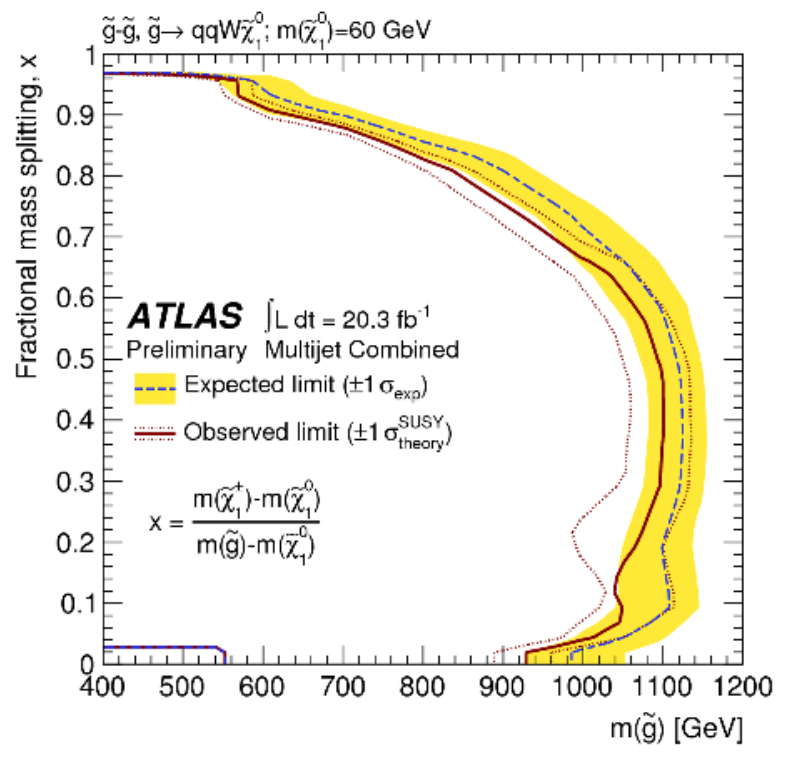

Figure 8. Exclusion limits of the ATLAS multijets analysis on gluino-pair production, with $\tilde{q} \rightarrow q \tilde{\chi}_{1}^{+}, \tilde{\chi}_{1}^{+} \rightarrow W^{+} \tilde{\chi}_{0}^{+}$. In the case investigated here, the limits are shown in the plane $x$ vs $m(\tilde{g})$, where $x$ is the ratio between the mass splitting of charginoneutralino over the mass splitting gluino-neutralino. The mass of the neutralino is set to $60 \mathrm{GeV}$.

the limits set by this analysis on the mSUGRA framework, with $\tan \beta=30$. Masses of gluinos around the $\mathrm{TeV}$ can be excluded for $m_{0}$ as high as $6 \mathrm{TeV}$. Fig. 8 shows the exclusions for this analysis in a simplified model where gluino pairs are produced, and each gluino decays to $q \bar{q} W \tilde{\chi}_{1}^{0}$ via an intermediate chargino.

\subsection{CMS: MET + b-jets}

CMS uses its full dataset $\left(19.4 \mathrm{fb}^{-1}\right)$ to search for SUSY signatures in fully hadronic events, with large $M E T$, at least three jets, at least one of which is tagged as a $b$ jet [10]. In order to be sensitive also to compressed spectra scenarios, a relatively loose selection on MET and $H T$ is performed: events with $M E T>125 \mathrm{GeV}$ and $H T>400$ $\mathrm{GeV}$ are selected and subdivided in four bins of $M E T$, four bins of $H T$ and three bins of $b$-jet multiplicity $(1,2$, or $\geq 3 b$-tagged jets in the event).

The analysis proceeds through a three-dimensional maximum likelihood fit, the three dimensions being $M E T$, $H T$, and the $b$-jet multiplicity. The dominant background arises from $t \bar{t}$, single $t$, and $W+$ jets events, followed by $Z \rightarrow v \bar{v}$ and QCD multijet events. These backgrounds are determined in a fully data-driven way, by using a single lepton (using $e$ 's and $\mu$ 's) control sample for the $t \bar{t}$, single $t$, and $W+$ jets background, $Z \rightarrow e^{+} e^{-}$and $Z \rightarrow \mu^{+} \mu^{-}$controls samples (with loosened $b$-tagging requirements) to estimate the $Z \rightarrow v \bar{v}$ component, and a sample enriched in QCD multijet events, obtained by reversing the cut on the minimum $\triangle \phi$ between $M E T$ and the three leading jets in the event. No assumption is made on the MET-HT shape of the backgrounds, rather it is taken from the data control samples. The potential contamination of signal events (as

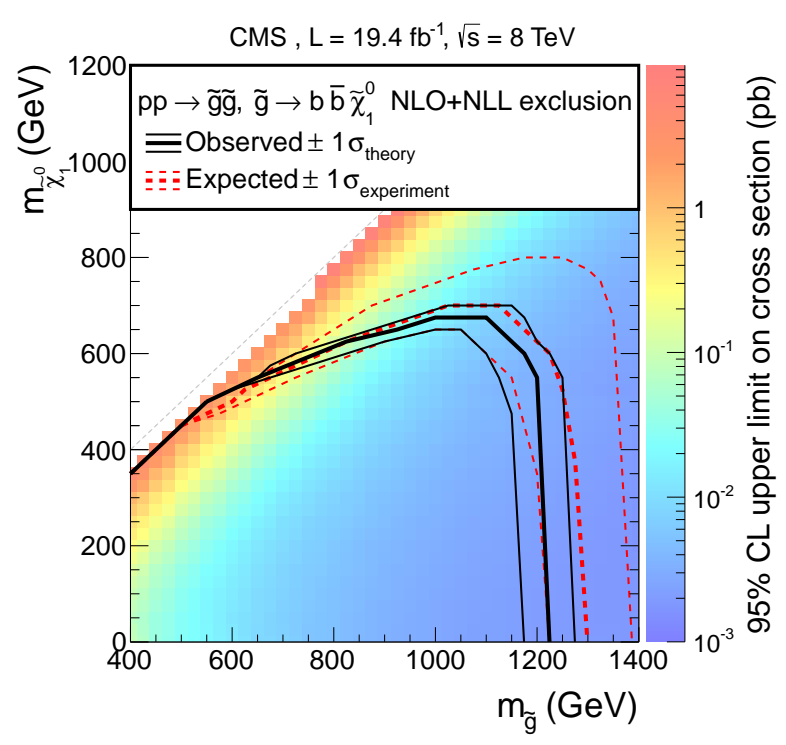

Figure 9. Exclusion limits for the CMS $M E T+b$-jets analysis on the simplified model in which gluino pairs are produced and each gluino decays to $b \bar{b} \tilde{\chi}_{1}^{0}$. The color scale indicates the observed cross-section 95\% CL upper limit, and the lines indicated the observed (black) and expected (red) exclusion limits.

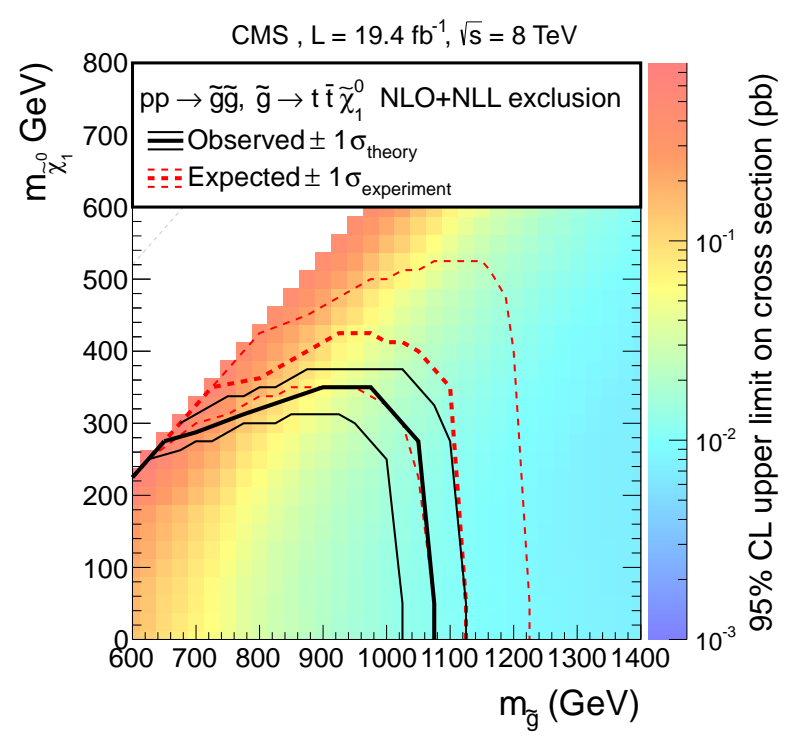

Figure 10. Exclusion limits for the CMS $M E T+b$-jets analysis on the simplified model in which gluino pairs are produced and each gluino decays to $t \bar{t} \tilde{\chi}_{1}^{0}$. The color scale indicates the observed cross-section 95\% CL upper limit, and the lines indicated the observed (black) and expected (red) exclusion limits.

predicted by the different SUSY models under investigation) into the control samples is taken into account in the fit.

No excess is observed, compared to the background predictions. Limits are set in the context of gluino-pair production, with each gluino decaying to $b \bar{b} \tilde{\chi}_{1}^{0}$ (see Fig. 9) or $t \bar{t} \tilde{\chi}_{1}^{0}$ (Fig. 10). The limits on the gluino mass, for a relatively light LSP, exceed $1 \mathrm{TeV}$ in both cases. 


\section{Conclusions}

The ATLAS and CMS experiments are performing a vast experimental campaign to detect signatures of physics beyond the standard model. The $2012 \sqrt{s}=8 \mathrm{TeV}$ dataset delivered by the LHC has already been extensively analyzed and more refined analyses are in the works. So far no statistically significant signal has been detected, and the limits on the masses of squarks and gluinos exceed the 1 $\mathrm{TeV}$ level in many of the scenarios investigated.

Additional interpretation plots for the analyses presented in this contribution are available from the public results pages of the ATLAS [11] and CMS [12] Collaborations.

The author would like to thank the SUSY Conveners of the ATLAS (Monica D'Onofrio and Andreas Hoecker) and of the CMS (Eva Halkiadakis and Frank Wuerthwein) experiments for the kind support given while preparing this talk.

\section{References}

[1] ATLAS Collaboration, Phys. Lett. B 716, 1 (2012); CMS Collaboration, Phys. Lett. B 716, 30 (2012).

[2] R. Barbieri, D. Pappadopulo, JHEP 0910, 61 (2009); M. Papucci, J. T. Ruderman, and A. Weiler, JHEP 1209, 35 (2012).

[3] ATLAS Collaboration, JINST 3, S08004 (2008); CMS Collaboration, JINST 3, S08004 (2008).

[4] CMS Collaboration, CMS-PAS-SUS-12-018.

[5] ATLAS Collaboration, ATLAS-CONF-2013-026.

[6] ATLAS Collaboration, ATLAS-CONF-2013-007.

[7] CMS Collaboration, arXiv: 1303.2985 [hep-ex], submitted to EPJC.

[8] ATLAS Collaboration, ATLAS-CONF-2013-047.

[9] ATLAS Collaboration, ATLAS-CONF-2013-054.

[10] CMS Collaboration, arXiv:1305.2390 [hep-ex], submitted to Phys. Lett. B.

[11] ATLAS Collaboration, https://twiki.cern.ch/twiki/ bin/view/AtlasPublic/SupersymmetryPublicResults.

[12] CMS Collaboration, https://twiki.cern.ch/twiki/bin/ view/CMSPublic/PhysicsResultsSUS. 